\title{
Solution structure of an intramembrane aspartyl protease by SANS
}

Raquel L. Lieberman ${ }^{1}$, Swe Htet Naing ${ }^{1}$, Ryan Oliver ${ }^{2}$,Kevin L. Weiss ${ }^{2}$, Volker Urban ${ }^{2}$. (1) School of Chemistry Biochemistry, Georgia Institute of Technology, Atlanta, Georgia, United States (2) MS-6460, Oak Ridge National Laboratory, Oak Ridge, Tennessee, United States

Email Contact: raquel.lieberman@chemistry.gatech.edu

Intramembrane aspartyl proteases (IAPs) comprise one of four families of integral membrane proteases that hydrolyze substrates within the hydrophobic lipid bilayer. IAPs include signal peptide peptidase, which processes remnant signal peptides from nascent polypeptides in the endoplasmic reticulum, and presenilin, the catalytic component of the $\gamma$-secretase complex that processes Notch and amyloid precursor protein. Despite their broad biomedical reach, basic structure-function relationships of IAPs remain active areas of research. Characterization of membrane-bound proteins is notoriously challenging due to their inherently hydrophobic character. For IAPs, oligomerization state in solution is one outstanding question, with previous proposals for monomer, dimer, tetramer, and octamer. Here we used small angle neutron scattering (SANS) to characterize n-dodecyl- $\beta$-D-maltopyranoside (DDM) detergent solutions containing and absent a microbial IAP ortholog. A unique feature of SANS is the ability to modulate the solvent composition to mask all but the enzyme of interest. The signal from the IAP was enhanced by deuteration and, uniquely, scattering from DDM and buffers were matched by the use of both tail-deuterated DDM and $\mathrm{D}_{2} \mathrm{O}$. Our study provides direct insight into the oligomeric state of purified IAP in surfactant solution, and demonstrates the utility of fully contrast-matching the detergent in SANS to characterize other intramembrane proteases and their membrane-bound substrates. 